\title{
Isolation and characterization of Faecalibacterium prausnitzii extracellular vesicles
}

\author{
Jafari $\mathbf{B}^{1}$, Khavari Nejad RA ${ }^{1}$, Vaziri $\mathbf{F}^{2}$, Siadat $\mathrm{SD}^{2^{*}}$ \\ ${ }^{1}$ Department of Biology, Science and Research Branch, Islamic Azad University, Tehran, Iran. \\ ${ }^{2}$ Department of Mycobacteriology and Pulmonary Research, Microbiology Research Center (MRC), Pasteur Institute of Iran, \\ Tehran, Iran.
}

\begin{abstract}
Introduction: Extracellular vesicles (EVs) contain active biological compounds which play important roles in biological processes. The secretion of EVs is a common phenomenon which occurs in archaea, bacteria and mammalian cells. The secretion of bacterial EVs has been discovered in various species of both Gram-negative and Gram-positive bacteria. Faecalibacterium prausnitzii is one of the commensal bacteria in human intestinal tract which has potentially therapeutic effects by secretion of bioactive compounds. Within the last few years, many investigations have been performed with respect to extracting and obtaining EVs and as a result, there are many methods to isolate and characterize EVs. The aim of this study was to isolate EVs from F. prausnitzii strain A2-165 and to characterize their physico-chemical properties. Methods: EVs were isolated from F.prausnitzii strain A2-165 using ultracentrifugation and filtration. The EVs of bacterium were then characterized by Scanning Electron Microscopy, SDS-PAGE, Bradford assay, NanoDrop and Limulus Amebocyte Lysate (LAL) test. Results: The extracted EVs were confirmed with the shape of vesicles and the sizes ranging from $\sim 30$ to $250 \mathrm{~nm}$. Total protein concentration of EVs were $\sim 3 \mathrm{mg} / \mathrm{ml}$ from two methods; Bradford and NanoDrop, respectively. Protein profile pattern of $F$. prausnitzii-derived EVs ranged from $11 \mathrm{up}$ to $245 \mathrm{kDa}$. Endotoxin measurement was $2.04 \mathrm{EU} / \mathrm{ml}$. Conclusion: The results of the current study demonstrated that EVs purity and conformation were acceptable. However, further investigations are necessary to elucidate the safety, efficacy, practicality and mechanism of action of this bacterium EVs in clinical practices, especially as vaccine delivery vehicles in the field of vaccine research.
\end{abstract}

KEYWORDS: Faecalibacterium prausnitzii, extracellular vesicles, isolation, characterization, vaccine vehicle.

\section{INTRODUCTION}

All domains of life and all cell types produce extracellular vesicles (EVs) [1]. Intracellular communication is an important feature of EVs which are membrane-enclosed vesicles released into the extracellular space. They are considered to play crucial roles in many physiological and pathological processes $[2,3]$. Recently, due to many researches in this field, many methods to isolate and characterize EVs, have been developed [4-6]. Bacteria release EVs ranging from 20-250 $\mathrm{nm}$ which are termed outer membrane vesicles (OMVs) and membrane vesicles (MVs) in Gram-negative and Gram-positive bacteria, respectively [7-10]. Bacterial EVs are small spherical structures, composed of cytosolic and membrane proteins, lipoproteins, phospholipids, glycolipids and nucleic acids [1116]. Meanwhile, EVs are generated in different bacterial growth phases and in various environments such as liquid, solid media and biofilm, as well as bacterial stress conditions [17-19].

*Corresponding Author: Seyed Davar Siadat, Department of Mycobacteriology and Pulmonary Research, Microbiology Research Center (MRC), Pasteur Institute of Iran, Tehran, Iran.

Email: d.siadat@gmail.com

Tel/Fax: (+98) 2164112823/ (+98) 2164112213
Currently, the use of bacterial EVs has gained attention due to their functional and practical roles in medical researches and their applications in new biotechnological tools such as vaccine manufacturing, adjuvants and drug delivery domains. They can also absorb anti-bacterial compounds such as antibiotics. Moreover, bacterial EVs play roles in host interactions, pathogenesis, quorum sensing and acquiring nutrition, to name a few [20].

EVs play interesting roles in human microbiota which have gained prominence in recent years. This is due to their ability in carrying an extensive range of enzymes used in digestion of polysaccharides, as well as their roles in immunomodulatory functions and signaling pathways [21]. One of the commensal bacteria in human gut is Faecalibacterium prausnitzii [22]. Several researches have indicated that one of the effective strategies used by $F$. prausnitzii is secretion of bioactive materials such as Microbial Anti-inflammatory Molecule (MAM) protein as well as salicylic, butyric and shikimic acids [23, 24]. Besides, Rossi et al. have isolated extracellular polymeric matrix (EPM) of $F$. prausnitzii strain HTF-F [25] which could be involved in many functional facets of the bacterium. Importantly, anti-inflammatory properties of 
secretions by this bacterium have been shown in some in vitro and in vivo studies [22-25]. The aim of this study was to isolate EVs from $F$. prausnitzii strain A2-165 and characterize their physico-chemical properties, as a first step for development of an EV-based vaccine delivery vehicle.

\section{MATERIALS and METHODS}

\section{Bacterial culture}

F. prausnitzii strain A2-165 (DSM $\mathrm{N}^{\circ}$ 17677) was purchased from DSMZ collection, Braunschweig, Germany and grown under anaerobic condition at $37^{\circ} \mathrm{C}$ [22].

\section{EVs isolation}

EVs of the bacterium were isolated using centrifugation and filtration protocols as described by Lee et al. [26], with some modifications. Briefly, 1 litre overnight culture of $F$. prausnitzii was centrifuged at $10,000 \times \mathrm{g}$ for $20 \mathrm{~min}$ at $4^{\circ} \mathrm{C}$, and the supernatant was filtered through a $0.45 \mu \mathrm{m}$ vacuum filter and then $0.22 \mu \mathrm{m}$ vacuum filter to remove the residual bacteria. The filtrate was ultracentrifuged at $200,000 \times \mathrm{g}$ for $2 \mathrm{~h}$ at $4^{\circ} \mathrm{C}$. The pellets were resuspended in one $\mathrm{ml}$ sucrose $(3 \%)$, and stored at $-80^{\circ} \mathrm{C}$ until use.

\section{EVs characterization}

\section{Scanning Electron Microscopy (SEM)}

SEM was used to confirm the integrity, stability and to determine the spatial shape and size of the vesicles. In this regard, filtered EVs in sucrose were covered on 400-mesh gold grids and stained with $2 \%$ uranyl acetate. To gain the image, a HITACHI S-4160 microscope (Nano-electronic Laboratory, Tehran University) was used.

\section{SDS-PAGE}

The proteins contents of the EVs were separated using SDSPAGE on $12 \%$ separating gel. The amount of $25 \mu \mathrm{l}$ of each sample was loaded and stained by Coomassie brilliant blue G250 dye.

Protein quantitation by NanoDrop and Bradford methods

To measure the concentration of the purified total protein, spectrophotometer NanoDrop Lite machine (Thermo scientific, USA) was used at the absorbance of $280 \mathrm{~nm}$. Bradford assay was performed according to its standard protocol using $100 \mu \mathrm{l}$ of protein and Bovine Serum Alboumin (BSA; $1 \mathrm{mg} / \mathrm{ml}$ ) as standard, read at $595 \mathrm{~nm}$.

\section{Endotoxin analysis by LAL method}

Lipopolysaccharide (LPS) was quantified by the chromogenic LAL assay. The process was carried out according to the manufacturer's instructions (Invitrogen, Carlsbad, CA, USA). The plate was read on EPSON LX-850 and the results were shown in endotoxin units (EU).

\section{RESULTS}

Isolation and characterization of EVs derived from $F$. prausnitzii strain A2-165

SEM confirmed the vesicular shape of the EVs, constituted of various sizes ranging from $\sim 30$ to $250 \mathrm{~nm}$ (Fig. 1).

The protein profile observed after SDS-PAGE showed bands ranging from 11 to $245 \mathrm{kDa}$ (Fig. 2). Total protein concentrations of the EVs were analyzed by NanoDrop and Bradford assays. The concentration of purified total protein was $\sim 3 \mathrm{mg} / \mathrm{ml}$ using Bradford assay which was confirmed by NanoDrop method as well. The amount of LPS, estimated by LAL method in the diluted sample was $2.04 \mathrm{EU} / \mathrm{ml}$.

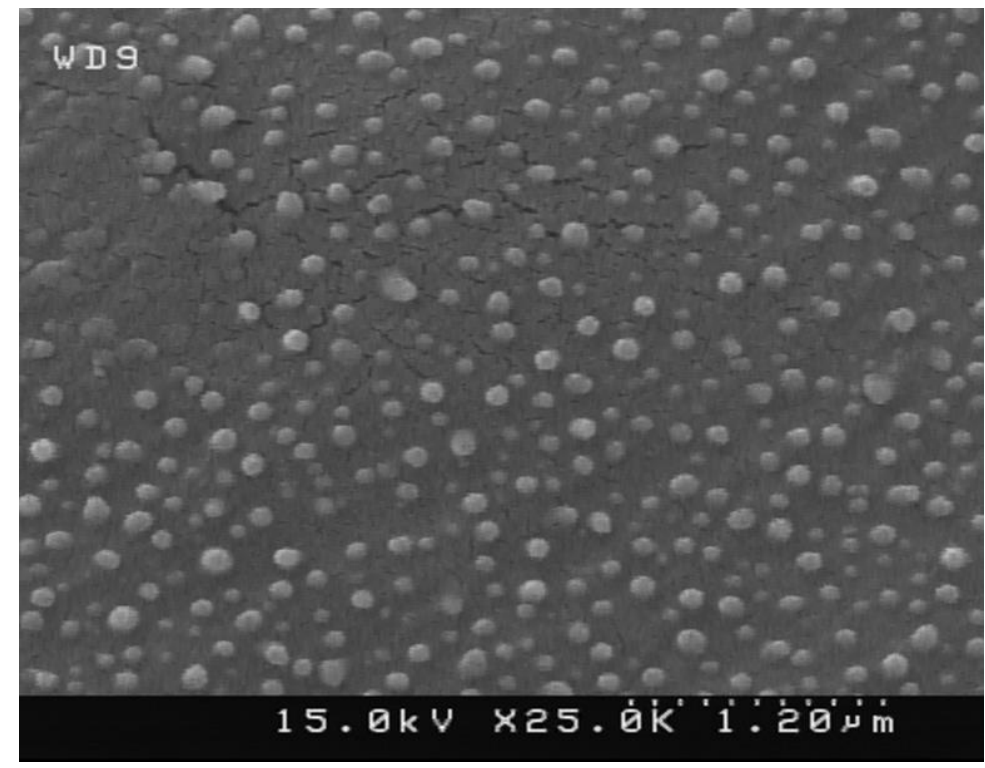

Fig. 1. SEM image of EVs isolated from F. prausnitzii.



Fig. 2. Protein profiles of EVs through SDS-PAGE.

\section{DISCUSSION}

In this study, we demonstrated that $F$. prausnitzii strain A2-165 which is one of the most important human gut microbiota, is able to produce and release EVs. Many methods have been developed for isolation and characterization of EVs in recent years. Hence, it could be assumed that different methods have direct effects on the downstream analyses [6]. Here, to isolate the EVs of $F$. prausnitzii, we performed ultracentrifugation method which is the most widely approach for this purpose [27]. Similar to our study, Chil-sung Kang et al. have also used ultracentrifugation at $200,000 \times \mathrm{g}$ for the isolation of Akkermansia muciniphila-derived EVs which is also a major bacterium in human gut microbiota [28]. 
In order to characterize EVs, the number of techniques could be various [4-6]. In this study, we used five of these techniques for characterization and further confirmation of $F$. prausnitzii secreted EVs. Gardiner et al. have reported that electron microscopy is among the three most widely used techniques for EV characterization [27]. In a research by Lee and co-workers, they have used Transmission Electron Microscopy (TEM) for the confirmation of Staphylococcus aureus-derived membrane vesicles and the measurement of MVs which was ranging from 20 to $100 \mathrm{~nm}$ [26]. We applied SEM for confirmation of spatial shape and size of $F$. prausnitzii-derived vesicles which their sizes were found to be within $\sim 30$ to $250 \mathrm{~nm}$ range.

Both Bradford and NanoDrop methods have been used as common protein assays used for EVs. Based on Gardiner's survey, protein assays for EV characterization have been performed by several scientists who have used Bradford assay and Nanodrop methods [27]. Haas et al. also used Bradford method to measure protein concentrations of the Streptococcus suis derived-EVs [29]. In our study, we used both techniques for measuring the total protein concentration of $F$. prausnitzii derived-EV. The results were $\sim 3$ and $3.307 \mathrm{mg} / \mathrm{ml}$ by Bradford and Nanodrop methods, respectively. SDS-PAGE is another method for EVs characterization [27]. Nevot et al. used Coomassie-stained SDS-PAGE (12\%) to show protein profile of Pseudoalteromonas antarctica-OMVs in which SDS-PAGE profiles revealed that the protein patterns of OMVs of this bacterium and its outer membrane are highly similar though not identical [30]. However in our research, the profile patterns of $F$. prausnitzii derived-EVs ranged from 11 to $245 \mathrm{kDa}$.

In our study, we used LAL assay for LPS measurement that read 2.04 EU/ml. However, analysis of this bacterial membrane suggests that this bacterium either lacks LPS or had an unusual structure [31]. The acceptable endotoxin level of this bacterium EVs in our study $(2.04 \mathrm{EU} / \mathrm{ml})$ which makes it a good candidate as a vaccine delivery vehicle [32].

In conclusion, our results demonstrated that EVs purity and conformation were within acceptable range. The identification of active components of $F$. prausnitzii secreted EVs and its potential therapeutic use remain to be investigated. Further researches are necessary to elucidate the safety, efficacy, practicality and mechanisms of action of this bacterial EVs in clinical practices, especially as vaccine delivery vehicles.

\section{ACKNOWLEDGEMENT}

We thank all the personnel of Mycobacteriology and Pulmonary Research Department, Pasteur Institute of Iran for their assistance in this project.

\section{CONFLICT OF INTEREST}

The authors declare that they have no conflict of interest.

\section{REFERENCES}

1. Brown L, Wolf JM, Prados-Rosales R, Casadevall A. Through the wall: extracellular vesicles in Gram-positive bacteria, mycobacteria and fungi. Nat Rev Microbiol. 2015;13(10):620-30. doi:10.1038/nrmicro3480.

2. Raposo G, Stoorvogel W. Extracellular vesicles: exosomes, microvesicles, and friends. J Cell Biol. 2013;200(4):373-83. doi: $10.1083 /$ jcb.201211138.

3. Yanez-Mo M, Siljander PR, Andreu Z, Zavec AB, Borras FE, Buzas EI et al. Biological properties of extracellular vesicles and their physiological functions. J Extracell Vesicles. 2015;4:27066. doi:10.3402/jev.v4.27066.
4. van der Pol E, Boing AN, Gool EL, Nieuwland R. Recent developments in the nomenclature, presence, isolation, detection and clinical impact of extracellular vesicles. J Thromb Haemost. 2016;14(1):48-56. doi:10.1111/jth.13190.

5. Gould SJ, Raposo G. As we wait: coping with an imperfect nomenclature for extracellular vesicles. J Extracell Vesicles. 2013;2. doi: $10.3402 /$ jev.v2i0.20389.

6. Lotvall J, Hill AF, Hochberg F, Buzas EI, Di Vizio D, Gardiner C et al. Minimal experimental requirements for definition of extracellular vesicles and their functions: a position statement from the International Society for Extracellular Vesicles. J Extracell Vesicles. 2014;3:26913. doi:10.3402/jev.v3.26913.

7. Kulkarni HM, Jagannadham MV. Biogenesis and multifaceted roles of outer membrane vesicles from Gram-negative bacteria. Microbiology. 2014;160(Pt 10):2109-21. doi:10.1099/mic.0.079400-0.

8. Baker JL, Chen L, Rosenthal JA, Putnam D, DeLisa MP. Microbial biosynthesis of designer outer membrane vesicles. Curr Opin Biotechnol. 2014;29:76-84. doi:10.1016/j.copbio.2014.02.018.

9. Mayrand D, Grenier D. Biological activities of outer membrane vesicles. Can J Microbiol. 1989;35(6):607-13.

10. Manning AJ, Kuehn MJ. Functional advantages conferred by extracellular prokaryotic membrane vesicles. J Mol Microbiol Biotechnol. 2013;23(1-2):131-41. doi:10.1159/000346548.

11. Yaron S, Kolling GL, Simon L, Matthews KR. Vesicle-mediated transfer of virulence genes from Escherichia coli O157:H7 to other enteric bacteria. Appl Environ Microbiol. 2000;66(10):4414-20.

12. Kadurugamuwa JL, Beveridge TJ. Virulence factors are released from Pseudomonas aeruginosa in association with membrane vesicles during normal growth and exposure to gentamicin: a novel mechanism of enzyme secretion. J Bacteriol. 1995;177(14):3998-4008.

13. van der Pol E, Boing AN, Harrison P, Sturk A, Nieuwland R. Classification, functions, and clinical relevance of extracellular vesicles. Pharmacol Rev. 2012;64(3):676-705. doi:10.1124/pr.112.005983.

14. Acevedo R, Fernandez S, Zayas C, Acosta A, Sarmiento ME, Ferro VA et al. Bacterial outer membrane vesicles and vaccine applications. Front Immunol. 2014;5:121. doi:10.3389/fimmu.2014.00121.

15. Rivera J, Cordero RJ, Nakouzi AS, Frases S, Nicola A, Casadevall A. Bacillus anthracis produces membrane-derived vesicles containing biologically active toxins. Proc Natl Acad Sci U S A. 2010;107(44):190027. doi:10.1073/pnas.1008843107.

16. Mashburn-Warren L, Mclean RJC, Whiteley M. Gram-negative outer membrane vesicles: beyond the cell surface. Geobiology. 2008;6(3):214-9. doi:10.1111/j.1472-4669.2008.00157.x.

17. Schwechheimer C, Kuehn MJ. Outer-membrane vesicles from Gramnegative bacteria: biogenesis and functions. Nat Rev Microbiol. 2015;13(10):605-19. doi:10.1038/nrmicro3525.

18. Kulp A, Kuehn MJ. Biological Functions and Biogenesis of Secreted Bacterial Outer Membrane Vesicles. Annu Rev Microbiol. 2010;64:163-84. doi:10.1146/annurev.micro.091208.073413.

19. Kuehn MJ. Secreted Bacterial Vesicles as Good Samaritans. Cell Host Microbe. 2012;12(4):392-3. doi:10.1016/j.chom.2012.10.005.

20. Fateh A, Vaziri F, Rahimi Janani F, Ahmadi Badi S, Ghazanfari M, Davari M, Arsang A, Siadat SD. New insight into the application of outer membrane vesicles of Gram negative bacteria. Vaccine Research. 2015;2(5). doi:10.18869/acadpub.vacres.2.5.93.

21. Shen Y, Giardino Torchia ML, Lawson GW, Karp CL, Ashwell JD, Mazmanian SK. Outer membrane vesicles of a human commensal mediate immune regulation and disease protection. Cell Host Microbe. 2012;12(4):509-20. doi:10.1016/j.chom.2012.08.004.

22. Sokol H, Pigneur B, Watterlot L, Lakhdari O, Bermudez-Humaran LG, Gratadoux JJ et al. Faecalibacterium prausnitzii is an anti-inflammatory commensal bacterium identified by gut microbiota analysis of Crohn disease patients. P Natl Acad Sci USA. 2008;105(43):16731-6. doi:10.1073/pnas.0804812105.

23. Quevrain E, Maubert MA, Michon C, Chain F, Marquant R, Tailhades J et al. Identification of an anti-inflammatory protein from Faecalibacterium prausnitzii, a commensal bacterium deficient in Crohn's disease. Gut. 2016;65(3):415-25. doi:10.1136/gutjnl-2014-307649.

24. Miquel S, Leclerc M, Martin R, Chain F, Lenoir M, Raguideau S et al. Identification of metabolic signatures linked to anti-inflammatory effects of Faecalibacterium prausnitzii. MBio. 2015;6(2). doi:10.1128/mBio.0030015.

25. Rossi O, Khan MT, Schwarzer M, Hudcovic T, Srutkova D, Duncan SH et al. Faecalibacterium prausnitzii Strain HTF-F and Its Extracellular Polymeric Matrix Attenuate Clinical Parameters in DSS-Induced Colitis. 
PLoS One. 2015;10(4):e0123013, doi:10.1371/journal.pone.0123013. 26. Lee EY, Choi DY, Kim DK, Kim JW, Park JO, Kim S et al. Grampositive bacteria produce membrane vesicles: proteomics-based characterization of Staphylococcus aureus-derived membrane vesicles. Proteomics. 2009;9(24):5425-36. doi:10.1002/pmic.200900338.

27. Gardiner C, Di Vizio D, Sahoo S, Thery C, Witwer KW, Wauben M et al. Techniques used for the isolation and characterization of extracellular vesicles: results of a worldwide survey. J Extracell Vesicles. 2016;5:32945. doi:10.3402/jev.v5.32945.

28. Kang CS, Ban M, Choi EJ, Moon HG, Jeon JS, Kim DK et al. Extracellular Vesicles Derived from Gut Microbiota, Especially Akkermansia muciniphila, Protect the Progression of Dextran Sulfate Sodium-Induced Colitis. Plos One. 2013;8(10). doi:ARTN e76520

10.1371/journal.pone.0076520.
29. Haas B, Grenier D. Isolation, Characterization and Biological Properties of Membrane Vesicles Produced by the Swine Pathogen Streptococcus suis. Plos One. 2015;10(6). doi:ARTN e013052810.1371/journal.pone.0130528.

30. Nevot M, Deroncele V, Messner P, Guinea J, Mercade E. Characterization of outer membrane vesicles released by the psychrotolerant bacterium Pseudoalteromonas antarctica NF3. Environ Microbiol. 2006;8(9):1523-33. doi:10.1111/j.1462-2920.2006.01043.x.

31. Miquel S, Martin R, Bridonneau C, Robert V, Sokol H, BermudezHumaran LG et al. Ecology and metabolism of the beneficial intestinal commensal bacterium Faecalibacterium prausnitzii. Gut Microbes. 2014;5(2):146-51. doi:10.4161/gmic.27651.

32. Brito LA, Singh M. Acceptable levels of endotoxin in vaccine formulations during preclinical research. J Pharm Sci. 2011;100(1):34-7. doi:10.1002/jps.22267. 